\title{
Shuttle walking test: a new approach for evaluating patients with pacemakers
}

\author{
Gillian E Payne, J Douglas Skehan
}

Abstract
Objective-To validate an incremental
field walking test, the shuttle walking test,
as a means of assessing pacing modes and
to aid programming of rate responsive
pacemakers.
Design-Three separate groups of patients were recruited. Reproducibility $(n=10)$ of the shuttle walking test was assessed by performing three consecutive tests. Comparison of the shuttle walking test with a 10 min walk was assessed in 20 patients. In the third group $(n=10)$ patients with rate responsive pacemakers were programmed to either VVI fixed rate 70 beats/min or VVIR with the optimal rate response to show the discriminative value of the test.

Setting-Pacing clinic in a regional cardiothoracic centre.

Patients-30 patients with chronotropic competence and dual chamber pacemakers with varying functional capacity and 10 patients with rate responsive pacemakers.

Interventions-Continuous haemodynamic monitoring was obtained using an ambulatory nuclear monitor, the Capintec VEST. Two exercise tests either shuttle walking test or $10 \mathrm{~min}$ corridor walk. The shuttle walk is an incremental walking test conducted on a $10 \mathrm{~m}$ course where the walking speed is dictated by bleeps on an audio cassette.

Results-Reproducibility was demonstrated over three consecutive tests with mean (1 SD) exercise times of $7.6(1 \cdot 7)$ min, $7.7(1.6)$, and $7.7(1.7) \mathrm{min}$. During the shuttle walk the test patients walked for a mean of $8.3(1.2)$ min producing peak relative cardiac outputs of $78(21)$ end diastolic volume/min compared with 64.9 (17) end diastolic volume/min for the 10 min walk $(P<0.001)$; peak heart rates were 118 and 104 beats/min $(P<0.03)$ respectively. In the third group relative peak cardiac output was significantly greater in VVIR (70 (24) $v$ VVI 52 (15) end diastolic volume/min) $(P<0.009)$ as were exercise times (VVIR $8 \cdot 8(1 \cdot 3) \min v$ VVI $8 \cdot 1(1 \cdot 3) \mathrm{min})(\mathrm{P}<0.003)$.

Conclusions-The shuttle walk is an easy test to administer, requiring little equipment. It produces a symptom limited maximal performance and will be a useful aid to pacemaker programming as it is reproducible and able to show differences in exercise capacity between pacing modes.

(Heart 1996;75:414-418)

Keywords: shuttle walking test; pacemaker programming

The ability to assess accurately the clinical capabilities of patients with pacemakers is of great value for the selection of optimal pacemaker variables. With the advent of rate responsive pacemakers, it is especially important to tailor the programming on an individual basis taking into account age, associated medical conditions, as well as functional capacity. ${ }^{1-3}$

The use of incremental treadmill exercise or a cycle ergometer is not always suitable because of age and severe underlying disease ${ }^{4}$ and failure to reproduce upper body movement, the stimulus to the activity sensor, in the same fashion as during everyday activities. ${ }^{5}$ This can lead to the sensor being set too sensitive for everyday requirements. Thus there is a need for a test that is simple to administer, with a minimum of specialist equipment and a good relation to the activities of daily living. The 6 or $10 \mathrm{~min}$ walking tests have been used but are difficult to standardise and easily influenced by encouragement from the supervising physician and patient motivation. ${ }^{6}$ In addition, their very simplicity often limits the information gained from them about the symptomatic changes that can occur with exercise. ${ }^{7}$

The shuttle walking test has been developed for the study of patients with chronic airways obstruction. ${ }^{8}$ It is a standardised incremental field walking test that provokes maximal performance and allows a direct comparison of a patient's performance. Its reproducibility has been demonstrated in patients with chronic obstructive airways disease. ${ }^{8}$ We report the characteristics of this type of ergometry in patients with pacemakers.

The aim of this study was to validate the shuttle walking test for use in patients with pacemakers and to show that it is a useful tool to aid assessment and programming of rate responsive pacemakers. 


\section{Patients and methods}

Evaluation of the shuttle walking test comprises four different studies to assess reproducibility, compare with more traditionally used methods, and discriminate between two pacing modes. All haemodynamic data for the study are provided by an ambulatory nuclear monitor, the Capintec VEST, ${ }^{9}$ which was originally used for the study of patients with ischaemic heart disease ${ }^{10}$ and has been shown by ourselves to be suitable for the study of patients with pacemakers. ${ }^{11}$ After an injection of technetium- $99 \mathrm{~m}$ a multigated acquisition scan is performed to allow correct positioning of the VEST and to help with calibration of the VEST. Details of how the VEST is positioned and functions are included in the appendix. As the data are stored on a Holter monitor and analysed off line the pacing mode can be blinded to the clinical observer as well as the patient.

All patients were recruited from the pacemaker clinic. Patients with a dual chamber pacemaker were considered eligible for the reproducibility and comparative studies, providing they were chronotropically competent. Patients were required to have rate responsive pacemakers for the discriminative study. Because of the small number of single chamber rate responsive units implanted at our centre all units used were dual chamber units. All patients were given a full explanation and written informed consent was obtained. As isotopes were involved patients were excluded if pregnant or breast feeding.

\section{SHUTTLE WALKING TEST}

This consists of a $10 \mathrm{~m}$ course marked out by two cones $9 \mathrm{~m}$ apart, to reduce the need for sudden changes in direction (fig 1). The speed is controlled by a series of bleeps delivered by an audio cassette, originally generated from a BBC microcomputer. The accuracy of the timed signal is maintained by inclusion of a calibration period of $1 \mathrm{~min}$ at the start of the tape. The test is preceded by a standardised explanation during which the patient is told to "walk at a steady pace, aiming to turn around when you hear the signal. You should continue to walk until you feel you are unable to maintain the required speed without becoming unduly breathless."

The start of the test is indicated by a triple bleep and each change in direction by a single bleep, so that by the time the patient heard the signal he should be rounding the cone to continue back down the course. Each minute the walking speed is increased by $0.17 \mathrm{~m} / \mathrm{s}$, so the subject is required to walk progressively faster. A change in speed is indicated by a triple bleep. There are 12 speeds or levels; the number of shuttles and the walking speed at that level is indicated in table 1 .

To help the patient establish the routine, for the first shuttle the clinical observer accompanied the patient. After that they had to coordinate their own speed with the timed signals. If the patient arrived at the cone before the bleep they were instructed to wait at the cone until the bleep sounded. The only instruction given by the observer was to remind the patient to increase his speed when the triple bleep sounded.

The end point of the test was reached either when the patient was too breathless to maintain the necessary speed or by the operator, if the patient failed to complete the shuttle in the time allowed, that is was greater than $0.5 \mathrm{~m}$ away from the cone when the bleep sounded.

\section{TEN MINUTE WALKING TEST}

The 10 min walking test was carried out on a $50 \mathrm{~m}$ hospital corridor. The patients were instructed to walk as far as they could during the $10 \mathrm{~min}$ period at their own speed with as many stops as they wanted. The operator was allowed only to inform the patient of the time remaining and could not provide any encouragement.

\section{REPRODUCIBILITY STUDY}

Ten patients performed three shuttle walking tests with $20 \mathrm{~min}$ rest between each test. Exercise times, peak heart rates, and relative cardiac outputs were recorded.

\section{COMPARATIVE STUDY}

Twenty patients were then randomised to either a shuttle walking test or a $10 \mathrm{~min}$ walk. After successful completion of the test the patients rested for $20 \mathrm{~min}$ before performing the second test. Exercise duration and peak cardiac output were recorded.

\section{DISCRIMINATION}

Ten patients with dual chamber rate responsive pacemakers were recruited. On the day of study they were programmed to VVI before performing the multigated acquisition scan about an hour before the first exercise test. It
Figure 1 The shuttle walk.

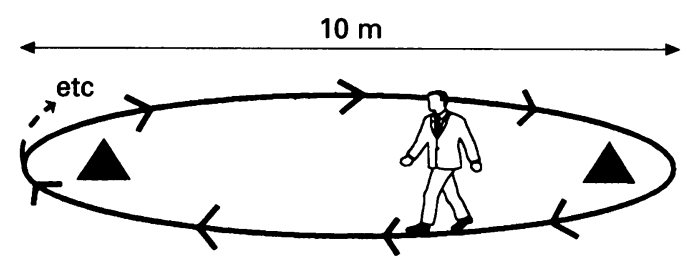

Audio cassette

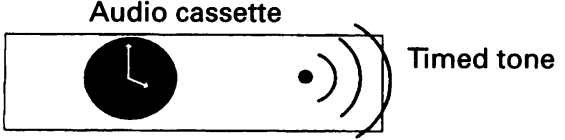

Table 1 Shuttle walk protocol

\begin{tabular}{cllc}
\hline Level & Speed $(\mathrm{m} / \mathrm{s})$ & Speed $(\mathrm{mph})$ & No of shuttles/ level \\
\hline 1 & 0.50 & 1.12 & 3 \\
2 & 0.67 & 1.50 & 4 \\
3 & 0.84 & 1.88 & 5 \\
4 & 1.01 & 2.26 & 6 \\
5 & 1.18 & 2.64 & 7 \\
6 & 1.35 & 3.02 & 8 \\
7 & 1.52 & 3.40 & 9 \\
8 & 1.69 & 3.78 & 10 \\
9 & 1.86 & 4.16 & 11 \\
10 & 2.03 & 4.54 & 12 \\
11 & 2.20 & 4.92 & 13 \\
12 & 2.37 & 5.30 & 14 \\
\hline
\end{tabular}


Table 2 Patient characteristics: reproducibility study

\begin{tabular}{llllll}
\hline Patient no & Age (years) & Sex & Heart disease & Ejection fraction (\%) & Pacemaker indication \\
\hline 1 & 60 & F & IHD & 62 & SND \\
2 & 70 & F & None & 47 & SND \\
3 & 66 & M & None & 54 & CHB \\
4 & 27 & M & None & 53 & SND \\
5 & 59 & N & None & 41 & CHD \\
6 & 76 & M & IHD & 34 & CHB \\
7 & 51 & F & None & 64 & CHB \\
8 & 59 & M & None & 46 & CHB \\
9 & 64 & F & None & 60 & CHB \\
10 & 74 & M & IHD & 35 & \\
\hline
\end{tabular}

CHB, Complete heart block; IHD, ischaemic heart disease; SND, sinus node disease.

\begin{tabular}{llllll} 
Table 3 & \multicolumn{7}{l}{ Patient characteristics: comparative study } \\
\hline Patient no & Age (years) & Sex & Heart disease & Ejection fraction (\%) & Echocardiogram abnormalities \\
\hline 1 & 60 & F & None & 62 & SND \\
2 & 41 & F & None & 65 & CHB \\
3 & 74 & M & IHD & 35 & CHB \\
4 & 27 & M & None & 53 & SND \\
5 & 45 & F & None & 70 & SND \\
6 & 68 & M & None & 46 & SND \\
7 & 62 & M & None & 62 & His ablation \\
8 & 66 & M & None & 43 & CHB \\
9 & 50 & F & None & 55 & SND \\
10 & 51 & $M$ & None & 52 & SND \\
11 & 58 & F & None & 49 & CHB \\
12 & 63 & $M$ & None & 52 & SND \\
13 & 76 & M IHD & 34 & SND \\
14 & 59 & M None & 41 & SNB \\
15 & 27 & M None & 53 & SND \\
16 & 66 & M N None & 54 & SND \\
17 & 70 & F & None & 47 & CHB \\
18 & 60 & F & IHD & 62 & SND \\
19 & 56 & M & None & 52 & \\
20 & 62 & F & None & 45 & \\
\hline
\end{tabular}

Abbreviations as given in table 2 .

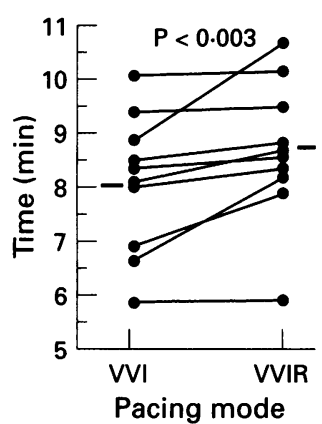

Figure 2 Exercise time during VVI and VVIR pacing. (Student's paired $t$ test.)

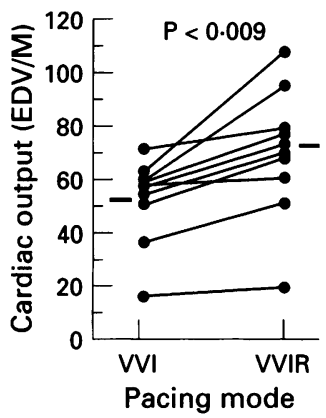

Figure 3 Relative cardiac output during VVI and VVIR pacing. EDVIM, end diastolic volumelmin. (Student's paired t test.) was felt that this test would have the greatest application in the programming of single chamber rate responsive pacemakers, thus VVI was compared with VVIR. Because of the small numbers of VVIR units implanted at our centre patients with DDDR units were used. They were randomised to either rate response as recommended by the manufacturer or a fixed rate of 70 beats $/ \mathrm{min}$. This was blinded to the patient and clinical observer. They performed one shuttle walking test in each mode with $20 \mathrm{~min}$ rest between.

\section{Results}

\section{REPRODUCIBILITY}

Good reproducibility of the shuttle walking test was shown by 10 patients who performed three consecutive walks (table 2). There was no significant difference in the duration of the three tests which was (mean (SD)) $7.6(1.7)$, $7.7(1.6)$, and $7.7(1.7) \mathrm{min}$. There was also no significant difference in the relative cardiac outputs generated by the VEST which were 74 (13), 75 (12), and 74 (13) end diastolic volume/min. This shows little or no learning phenomenon and good reproducibility.

\section{COMPARISONS}

All 20 patients performing the shuttle walking test and the 10 min walk found both tests easy to perform (table 3). During the shuttle test patients walked for a mean (1 SD) (range) of $8.3(1 \cdot 2)(6-10 \cdot 3) \mathrm{min}$ producing mean (1 SD) (range) peak relative cardiac outputs of 78 (21) (42-106) end diastolic volume/min compared with 64.9 (17) (35-89) end diastolic volume/min for the $10 \mathrm{~min}$ walk, which is significantly greater $(\mathbf{P}<0.001)$ (paired $t$ test).
Peak heart rates during the shuttle walk were $118 \mathrm{beats} / \mathrm{min}$ and during the $10 \mathrm{~min}$ walk 104 beats/min $(P<0.03)$, maximum speeds were mean (1 SD) $5.8(0.7)$ and $4.6(0.8) \mathrm{m} / \mathrm{s}$ $(\mathrm{P}<0.05)$, respectively.

\section{DISCRIMINATION}

Ten patients with rate responsive pacemaker performed two exercise tests (table 4). Exercise time and peak relative cardiac output were significantly greater in VVIR than VVI (VVIR $8.8(1.3) \quad v$ VVI $8.1(1.3) \mathrm{min}$, $\mathrm{P}<0.003$ (fig 2) and VVIR $70(24) v$ VVI 52 (15) end diastolic volume $/ \mathrm{min}, \quad P<0.009$ Student's $t$ test (fig 3)).

\section{Discussion}

The advent of rate responsive pacing has meant that some form of exercise testing is essential for optimal pacemaker programming. ${ }^{212}{ }^{13}$ The shuttle walking test can be easily performed within a short period of time without technical equipment. The external speed control helps reduce operator bias. Each patient receives the same instructions each time that he or she performs the test and although the clinical observer is present and walks with the patient during the first minute, the external speed control prevents the patient walking any faster and is essential to prevent the more ambulant patients starting off too quickly. This test produces reproducible results which show little or no learning effect and apply irrespective of whether the patient completes one or all 12 levels. The test requires no expensive equipment and is ideal for use in the setting of a pacemaker clinic. The 6 or 10 min walk has often been used for the assessment of patients with pacemakers. Although this is easy to implement, it is easily influenced by external factors, ${ }^{6}$ which make it difficult to reproduce the same test even within the same patient. External speed control as with the shuttle walk permits valid interpatient and intrapatient comparison as at a certain distance along the course each patient will have experienced similar "work rate/stress". This is not always the case for patients completing similar distances on a self paced walking test and the habitual nature of a self paced test may prevent such a test showing any improvement from pacemaker reprogramming. ${ }^{14}$

The assessment of day to day activities has shown that they are usually of an irregular nature and a steady state is rarely achieved. ${ }^{15}$ Consequently, patients symptoms are probably more likely to be identified during an incremental test rather than a self paced exercise test. The $10 \mathrm{~min}$ walk has no set pace and whether the cardiorespiratory system is stressed maximally depends on how the test is conducted. The lack of external encouragement during this walk means that the result is submaximal and this is reflected by the significantly lower cardiac output and heart rate than observed in the shuttle walking test. This means that as a discriminative test it is of little value, as reflected in the variance in relative 
Table 4 Patient characteristics: discriminate study

\begin{tabular}{|c|c|c|c|c|c|c|}
\hline$\overline{\text { Patient no }}$ & Age (years) & Sex & Heart disease & Ejection fraction (\%) & Pacemaker indication & Pacemaker \\
\hline 1 & 74 & $\mathbf{M}$ & IHD & 35 & $\mathrm{CHB}$ & Elite \\
\hline 2 & 66 & M & None & 54 & $\mathrm{CHB}$ & Thera VDD \\
\hline 3 & 49 & $\mathbf{M}$ & None & 41 & SND & Vigor \\
\hline 4 & 35 & M & None & 43 & $\mathrm{CHB}$ & Chorus RM \\
\hline 5 & 64 & $\mathrm{~F}$ & None & 55 & $\mathrm{CHB}$ & Elite \\
\hline 6 & 42 & $\mathrm{~F}$ & None & 60 & $\mathrm{CHB}$ & Elite \\
\hline 7 & 65 & M & IHD & 19 & $\mathrm{CHB}$ & Chorus RM \\
\hline 8 & 35 & $\mathrm{~F}$ & Dextrocardia & 38 & $\mathrm{CHB}$ & Vigor \\
\hline 9 & 62 & $M$ & None & 55 & $\mathrm{CHB}$ & Chorus RM \\
\hline 10 & 67 & M & IHD & 43 & $\mathrm{CHB}$ & Elite \\
\hline
\end{tabular}

Abbreviations as given in table 2 .

cardiac outputs for the two tests (432 for the shuttle walk and 303 for the 10 min walk).

Incremental treadmill exercise is a particularly unfamiliar and potentially intimidating test. Repeat testing is recognised to involve a learning effect and may prevent accurate comparison. The treadmill has been over used and there is a real need for a test which is simple and involves an everyday activity. The shuttle walk is based on a familiar activity, providing a better physiological response and if repeat tests are necessary (as is often the case when programming rate responsive pacemakers) then it reduces the limitations on activity from muscle fatigue.

The graded nature of the shuttle walking test is shown by the incremental heart rate response which is not present during the 10 min walk. Typical plots (fig 4 ) of the heart rate responses during the $10 \mathrm{~min}$ walk and the shuttle test show that with the former the patient very rapidly reaches an equilibrium. The shuttle walking test also requires less space than the 10 min walk.

To aid programming of rate responsive pacemakers a mean heart rate after each minute was calculated. However, there was such wide deviation in heart rate responses in a patient population with theoretically normal rate response that this was not felt to be valuable. This might be expected from the varying ages involved in this study but there was no correlation of predicted peak heart rate $(220$ beats/min-age) with either the heart rate after $5 \mathrm{~min}$ or the peak heart rate. This highlights the inherent problem with programming rate responsive pacemakers, which is that it is impossible to determine the "optimal rate" for every patient for a given level of exercise by arbitrary means. Research should be directed towards the development of a sensor capable

Figure 4 Typical heart rate responses during the shuttle and corridor walks.

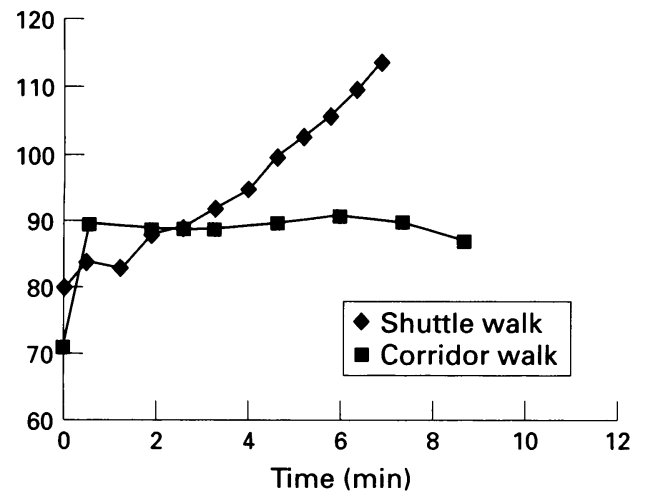

of optimal rate determination and a shuttle test can provide a valuable assessment of such development.

In conclusion this test produces symptom limited maximal exercise, is easy for patients to perform, reproducible and permits fair comparison of sensors.

1 Clarke M, Sutton R, Ward D, Camm AJ, Rickards A, Ingram A, et al. Recommendations for pacemaker prescription for symptomatic bradycardia. Br Heart $\mathcal{F} 1991$; 66: 185-91.

2 Sowton E. Exercise testing in the indication and evaluation of pacemaker treatment. Eur Heart f 1987;8:155-8.

Lau CP. The range of sensors and algorithms used in rate adaptive cardiac pacing. PACE 1992;15:1177-211.

4 Langenfeld H, Schneider B, Grimm W, et al. The six minute walk an adequate exercise test for patients? $P A C E$ minute walk an adeq

5 Provenier F, Jordaens L. Evaluation of six minute walking test in patients with single chamber rate responsive pacemakers. Br Heart $\mathcal{F}$ 1994;72:192-6.

6 Guyatt GH, Pugsley SO, Sullivan MJ, Thompson PJ, Berman LB, Jones NL, et al. Effect of encouragement on walking test performance. Thorax 1984;39:818-22.

7 Beaumont A, Crockcroft A, Guz A. A self paced treadmill walking test for breathless patients. Thorax 1985;40: 459-64

8 Singh SJ, Morgan MDL, Scott S, Walters D, Hardman E. Development of a shuttle walking test of disability in patients with chronic airways obstruction. Thorax 1992;47:1019-24

9 Wilson RA, Sullivan PJ, Moore RH, Zielonka JS, Alpert $\mathrm{NM}$, Boucher CA, et al. An ambulatory ventricular function monitor: validation and preliminary results. $A m \mathcal{F}$ Cardiol 1983;52:601-6.

10 Gold HK, Strauss HW. Continuous measurement of left ventricular function by an ambulatory monitor in patients with coronary disease. $7 \mathrm{Am}$ Coll Cardiol 1988; 12:669-79.

11 Payne GE, Williams $\mathrm{H}$, Skehan JD. A new approach in the assessment of pacing haemodynamics: a comparison of DDD and VVI. PACE 1995 Oct.

12 Furman S. Rate modulated pacing. Circulation 1990;82: 1081-94.

13 Sulke N, Dritsas A, Chambers J, Sowton E. Is accurate rate response programming necessary? PACE 1990;13: 1031-44.

14 Swinburn CR, Wakefield JM, Jones PW. Performance, ventilation, and oxygen consumption in three different types of exercise test in patients with chronic obstructive lung disease. Thorax 1985;40:581-6.

15 Spiro SG. Exercise testing in clinical medicine. $\mathrm{Br} F \mathrm{Dis}$ Chest 1977;71:145-72.

\section{Appendix}

The detector placed over the left ventricle consists of a $5.6 \mathrm{~cm}$ diameter sodium iodide crystal mated to two photo cubes. The detector views the left ventricle through a parallel hole colliminator. Energy discrimination is achieved with a thresholding circuit set to accept events of greater than $120 \mathrm{kev}$. The digital data from the thresholding circuit are averaged for $31.25 \mathrm{~ms}$, converted to an analogue signal, and recorded simultaneously with the electrocardiogram.

Before a VEST study, patients have a multigated equilibrium blood pool scan at rest for baseline definition of global and regional left ventricular functions. Radionuclide ventriculography is performed after red blood cell labelling using intravenous injection of 20 $\mathrm{mCi}$ of $99 \mathrm{mTC}$ pertechnetate. Images are recorded in the anterior view in the supine position and the left anterior oblique view in the sitting position (about 10 $\mathrm{min} / \mathrm{view}$ ) with a general all purpose parallel hole collimator. The left ventricular time activity curves are generated after background correction and the left ventricular ejection fraction is calculated manually three times and the mean taken. This has been found to be more consistent than early versions for automatic calculation. These data are then used for subsequent comparison with the VEST data.

On completion of their gated scan, electrodes are placed in the CM5 and CC 5 position and the patient asked to resume the sitting position. The plastic garment (VEST) is then fitted to the patient's thorax and tightened into place. There are three different sizes of vest to allow a snug fit. A targeting device is employed to ensure correct positioning of the detector over the left ventricle. The left anterior oblique view is chosen as this shows the interventricular septum, thereby opti- 
mising separation of the right and left ventricular blood pools. When the best position is produced the garment is tightened. The detector is then screwed into place and a static image taken to record its correct position. The detector and the electrocardiograph leads are connected to a tape recorder carried over the shoulder. The left ventricle detector weighs $1.5 \mathrm{lb}(0.675 \mathrm{~kg})$ with the entire unit weighing $7.5 \mathrm{lb}(3.375 \mathrm{~kg})$.

The radionuclide and electrocardiographic data are then processed on IBM computer software provided by the manufacturer. Unfortunately, the software has a problem with identifying the paced $R$ waves, particularly for unipolar pacing spikes. It is therefore necessary to perform beat by beat review of the data to ensure correct $\mathbf{R}$ wave identification. It is also possible to exclude poor quality data.

The baseline data on ejection fraction from the initial scan are used to calculate background scatter correction. The acquired data are then analysed for heart rate, ejection fraction, filling rate, end diastolic and end systolic volumes, and relative cardiac output. The data are the summation of 30 beats to create an average time activity curve. The left ventricular ejection is calculated in a standard fashion (end diastolic counts minus end systolic counts minus background corrected end diastolic counts). 